\title{
Does solar physics provide constraints to weakly interacting massive particles?
}

\author{
A. Bottino, ${ }^{1, *}$ G. Fiorentini, ${ }^{2, \dagger}$ N. Fornengo, ${ }^{1, \hbar}$ B. Ricci, ${ }^{2, \S}$ S. Scopel, ${ }^{1, \|}$ and F. L. Villante ${ }^{2, \mathbb{I}}$ \\ ${ }^{1}$ Dipartimento di Fisica Teorica, Università di Torino Istituto Nazionale di Fisica Nucleare, Sezione di Torino via P. Giuria 1, \\ I-10125 Torino, Italy \\ ${ }^{2}$ Dipartimento di Fisica, Università di Ferrara, Istituto Nazionale di Fisica Nucleare, Sezione di Ferrara via del Paradiso 12, \\ I-44100 Ferrara, Italy
}

(Received 26 June 2002; published 23 September 2002)

\begin{abstract}
We investigate whether present data on helioseismology and solar neutrino fluxes may constrain weakly interacting massive particle (WIMP)-matter interactions in the range of WIMP parameters under current exploration in WIMP searches. We find that, for a WIMP mass of $30 \mathrm{GeV}$, once the effect of the presence of WIMPs in the Sun's interior is maximized, the squared isothermal sound speed is modified, with respect to the standard solar model, by at most $0.4 \%$ at the Sun's center. The maximal effect on the ${ }^{8} \mathrm{~B}$ solar neutrino flux is a reduction of $4.5 \%$. Larger masses lead to smaller effects. These results imply that present sensitivities in the measurements of solar properties, though greatly improved in recent years, do not provide information or constraints on WIMP properties of relevance for dark matter. Furthermore, we show that, when current bounds from direct WIMP searches are taken into account, the effect induced by WIMPs with dominant coherent interactions is drastically reduced as compared to the values quoted above. The case of neutralinos in the minimal supersymmetric standard model is also discussed.
\end{abstract}

DOI: 10.1103/PhysRevD.66.053005

PACS number(s): 95.35.+d, 98.35.Gi, 98.62.Gq

\section{INTRODUCTION}

A host of independent astronomical observations point to the existence in our Universe of a total amount of matter in the range $0.2 \leq \Omega_{m} \leq 0.4$ [1] (or equivalently, $0.05 \leqq \Omega_{m} h^{2}$ $\lesssim 0.3$, where $h$ is the Hubble constant in units of $100 \mathrm{~km} \mathrm{~s}^{-1} \mathrm{Mpc}^{-1}$ ), well beyond the amount of visible matter $\Omega_{v i s} \sim 0.003$. Since the primordial nucleosynthesis tells us that baryons cannot contribute for more than about $5 \%$ [2], most of the dark matter must be nonbaryonic. The evolution theory of the primordial density fluctuations into the present cosmological structures indicates that most of the dark matter must be comprised of cold particles, i.e., of particles that decoupled from the primordial plasma when nonrelativistic. A particle with the suitable properties for being a significant cold dark matter relic is generically defined as a WIMP (weakly interacting massive particle).

A variety of physical realizations for a WIMP are offered by various extensions of the standard model [3], the neutralino being one of the most appealing candidates. In the present paper, many considerations are developed in terms of generic WIMPs. We consider the neutralino, whenever we wish to narrow down to a specific candidate.

WIMPs are very actively searched for by means of various experimental strategies. Direct searches rely on the measurements of the signal that a nucleus of an appropriate detector would generate, when hit by a WIMP [4]. Indirect

\footnotetext{
*Electronic address: bottino@to.infn.it

†Electronic address: fiorentini@fe.infn.it

*Electronic address: fornengo@to.infn.it; URL: http://www.to.infn.it/ fornengo

${ }^{\S}$ Electronic address: ricci@fe.infn.it

"Electronic address: scopel@to.infn.it

II Electronic address: villante@fe.infn.it
}

searches are based on measurements of the signals due to WIMP pair annihilations in the galactic halo or inside celestial bodies [3].

The event rates of WIMP direct search experiments are proportional to the product of the local WIMP density $\rho_{\chi}$ in the Galaxy times the WIMP-nucleus cross section. In what follows $\rho_{\chi}$ will be expressed as $\rho_{\chi}=\xi \cdot \rho_{l}$, where $\rho_{l}$ is the local total dark matter density and $\xi(\xi \leqslant 1)$ is a scaling parameter which accounts for the actual fraction of local dark matter to be ascribed to the candidate $\chi$.

WIMP-matter interactions are conveniently classified in terms of coherent cross sections and (nuclear) spindependent ones. In the first case, the WIMP-nucleus cross section is given in terms of the WIMP-proton and WIMPneutron cross sections. To simplify the formalism, in the following we further assume that, in the coherent case, WIMPs interact with equal strength with protons and neutrons (for instance, this may safely be assumed for neutralinos, while it is not the case for neutrinos), and then we express a generic WIMP-nucleus coherent cross section in terms of a single WIMP-nucleon cross section $\sigma_{c}$. For the spin-dependent case, the derivation of a WIMP-nucleon cross section from the WIMP-nuclear one depends on the nature of the WIMP and on specific nuclear properties [5].

In the case of WIMPs with coherent interactions with matter, the range of WIMP parameters under current exploration in WIMP direct searches is conveniently expressed in terms of the quantity $\xi \sigma_{c}$ :

$$
10^{-43} \mathrm{~cm}^{2} \leqslant \xi \sigma_{c} \leqslant 6 \times 10^{-41} \mathrm{~cm}^{2},
$$

for WIMP masses $m_{\chi}$ in the range

$$
30 \mathrm{GeV} \leqslant m_{\chi} \leqslant 270 \mathrm{GeV} \text {. }
$$

In the derivation of the ranges in Eqs. (1), (2), one has taken into account a variety of WIMP distribution functions 
in the galactic halo and uncertainties in the determination of the relevant astrophysical quantities $[6,7]$.

For the reasons mentioned above, in the case of spindependent interactions no model-independent sensitivity range may be derived. However, to give an indication, we recall that, in the case of a relic particle which interacts with matter mainly by spin-dependent interactions mediated by $Z$-boson exchange, current WIMP direct experiments are sensitive to values of $\xi \sigma_{s d}$ of about $10^{-36}-10^{-37} \mathrm{~cm}^{2}$ [5], where $\sigma_{s d}$ is the spin-dependent WIMP-proton cross section.

One of the WIMP direct search experiments, the DAMA/ $\mathrm{NaI}(\mathrm{Tl})$ experiment [8], has observed an annual modulation effect (at a $4 \sigma$ C.L.) with all the features expected for a WIMP signal [9]. When interpreted as due to a WIMP with coherent interactions, the DAMA effect provides a $3 \sigma$ region in the plane $m_{\chi^{-}} \xi \sigma_{c}$ embedded in the range of Eqs. (1), (2). In Ref. [10] it was proved that this annual modulation effect is compatible with an interpretation in terms of relic neutralinos (see also Ref. [11]).

If WIMPs populate our Galaxy, they can be captured by the Sun and accumulate in its core. For suitable values of the WIMP parameters, this could have relevant effects on the Sun. In fact, WIMPs could provide an effective mechanism for energy transport in the Sun, producing an isothermal core and reducing substantially the Sun central temperature $T_{c}$. Following this idea, some time ago a special class of WIMPs named cosmions, with masses of a few $\mathrm{GeV}$ and scattering cross sections on nucleons of the order of $10^{-36}-10^{-34} \mathrm{~cm}^{2}$, was studied in detail as a way of solving simultaneously the solar neutrino puzzle and the dark matter problem (see e.g., Refs. [12-17]). The cosmion hypothesis was progressively abandoned when it became clear that the solar neutrino puzzle cannot be accounted for by simply reducing $T_{c}$.

In the last twenty years, our observational knowledge of the solar interior has progressed enormously. By means of helioseismic data it has become possible to derive the sound speed with an accuracy of about one part per thousand over most of the radial profile and of about one percent in the innermost part [18]. By the same method, it has been possible to deduce important properties of the convective envelope. The photospheric helium fraction $Y_{p h}$ and the depth of the convective envelope $R_{b}$ have been determined with an accuracy of about one percent and one per thousand respectively, following the pioneering papers of Refs. [19,20]. Moreover, the measurement of the neutrino flux from ${ }^{8} \mathrm{~B}$ decay obtained by combining [21-24] SNO charged current [25] and super-Kamiokande [26] data and confirmed by the recent SNO neutral current results [27], has provided a determination of the temperature $T_{c}$ near the center of the Sun at the level of about one percent [28].

All the predictions of the standard solar model (SSM) have been confirmed by these accurate tests, so one is naturally led to the question of whether our accurate knowledge of the Sun interior can be used to constrain the WIMP parameter space. This particularly interesting question was recently raised in Refs. [29] and [30], where it was concluded that solar physics can be used to significantly constrain the WIMP parameter space.
In this paper, we provide an alternative analysis of the problem, obtaining results substantially different from those derived in Refs. [29,30].

The plan of the paper is as follows. In Sec. II we discuss WIMP energy transport in the Sun, introducing the relevant physical parameters to be discussed in the subsequent sections. In Secs. III and IV WIMP energy transport is applied to the central solar structure, in order to determine the regions of the WIMP parameter space where the WIMP energy transport is most efficient. This leads to an optimal choice of WIMP parameters which is then used in Sec. V to calculate the solar sound speed profile and discuss the possible impact of helioseismology on WIMP properties. The sensitivity of the available data on ${ }^{8} \mathrm{~B}$ solar neutrinos to the presence of WIMPs in the Sun is then discussed in Sec. VI. The neutralino, as a specific realization of WIMP, is discussed in Sec. VII. Section VIII is devoted to our conclusions.

\section{WIMP ENERGY TRANSPORT}

In this section, we briefly review some issues relevant to the problem of WIMP energy transport in the Sun addressed in Refs. [12-17].

WIMPs are captured by the Sun during the course of its lifetime and are confined by the effect of gravity in a central region with a radial number density distribution approximately given by $[31,32]$ (we hereby use natural units: $\hbar=c$ $=k_{B}=1$ )

$$
n_{\chi}(r)=\left(n_{\chi}\right)_{c} \exp \left(-\frac{r^{2}}{r_{\chi}^{2}}\right),
$$

where the quantity $\left(n_{\chi}\right)_{c}=\left(1 / \pi^{3 / 2}\right)\left(N_{\chi} / r_{\chi}^{3}\right)$ is the central WIMP number density, $N_{\chi}$ is the total number of WIMPs in the Sun and

$$
r_{\chi}=\left(\frac{3 T_{c}}{2 \pi G \rho_{c} m_{\chi}}\right)^{1 / 2},
$$

$T_{c}$ and $\rho_{c}$ being the Sun central temperature and density, and $m_{\chi}$ the WIMP mass. For $T_{c}=1.57 \times 10^{7} \mathrm{~K}$ and $\rho_{c}$ $=154 \mathrm{~g} \mathrm{~cm}^{-3}$, one finds $r_{\chi} \simeq 0.01\left(100 \mathrm{GeV} / m_{\chi}\right)^{1 / 2} R_{\odot}$. We note that Eq. (3) is obtained under the assumption of constant density and temperature in the region of the Sun populated by WIMPs. This approximation is accurate enough for our purposes.

WIMPs provide a mechanism for energy transport in the solar core whose efficiency depends on the Knudsen parameter

$$
K=\frac{l_{\chi}(0)}{r_{\chi}}
$$

where $l_{\chi}(0)$ is the WIMP mean free path near the solar center and generally

$$
\frac{1}{l_{\chi}(r)}=\sum_{i} \sigma_{i} X_{i}(r) \frac{\rho(r)}{m_{i}} .
$$


Here the sum extends to all the nuclear species present in the Sun, $X_{i}$ is the mass fraction of the $i$ th element, $\rho(r)$ is the density profile of the Sun and $\sigma_{i}$ is the WIMP scattering cross section with the nucleus of species $i$.

For $K \ll 1$ the WIMP mean free path is much smaller than the dimension of the region where the WIMPs are confined and the energy transfer mechanism is conductive. The problem of thermal conduction by a dilute gas of massive particles is discussed in Ref. [12]. For our purposes, it is useful to express the WIMP luminosity in the conductive regime by using the radiative transport equation:

$$
L_{\chi, \text { cond }}(r)=-\frac{16 \pi a_{\mathrm{rad}}}{3} \frac{T(r)^{3} r^{2}}{\kappa_{\chi}(r) \rho(r)} \frac{d T}{d r},
$$

where the WIMP opacity $\kappa_{\chi}$ is defined as

$$
\frac{1}{\kappa_{\chi}(r)}=\frac{3 n_{\chi}(r)}{4 a_{\mathrm{rad}} T(r)^{3}} \sqrt{\frac{T(r)}{m_{\chi}}} \rho(r) l_{\chi}(r) \lambda_{\chi} .
$$

In Eqs. (7), (8) the quantity $a_{\text {rad }}$ denotes the radiation density constant and $\lambda_{\chi}$ is a dimensionless coefficient which depends on the ratio between the WIMP mass and the masses of background nuclei. ${ }^{1}$

For $K \gg 1$ the WIMP mean free path is much larger than the region in which WIMPs are trapped and the energy transfer mechanism is nonlocal. An analytic approximation to treat this case has been developed by Spergel and Press [13] who described WIMPs as an isothermal gas at an appropriate temperature $T_{\chi}$. In this case, the luminosity $L_{\chi, \mathrm{NL}}$ carried by WIMPs can be expressed as

$$
L_{\chi, \mathrm{NL}}(r)=\int_{0}^{r} d r^{\prime} 4 \pi r^{\prime 2} \rho\left(r^{\prime}\right) \epsilon_{\mathrm{SP}}\left(r^{\prime} ; T_{\chi}\right),
$$

where $\epsilon_{\mathrm{SP}}$ represents the energy transferred to WIMPs per second and per gram of nuclear matter and is given by

$$
\begin{aligned}
& \epsilon_{\mathrm{SP}}\left(r ; T_{\chi}\right)=8 \sqrt{\frac{2}{\pi}} n_{\chi}(r) \sum_{i} \sigma_{i} \frac{X_{i}(r)}{m_{i}} \frac{m_{\chi} m_{i}}{\left(m_{\chi}+m_{i}\right)^{2}} \\
& \times\left(\frac{m_{\chi} T(r)+m_{i} T_{\chi}}{m_{\chi} m_{i}}\right)^{1 / 2}\left[T(r)-T_{\chi}\right] .
\end{aligned}
$$

The WIMP temperature $T_{\chi}$ is fixed by requiring that the energy absorbed by WIMPs in the inner region [where $\left.T(r)>T_{\chi}\right]$ is equal to that released in the outer region [where $\left.T(r)<T_{\chi}\right]$ : i.e.,

$$
L_{\chi, \mathrm{NL}}\left(R_{\odot}\right)=\int_{0}^{R_{\odot}} d r^{\prime} 4 \pi r^{\prime 2} \rho\left(r^{\prime}\right) \epsilon_{\mathrm{SP}}\left(r^{\prime} ; T_{\chi}\right)=0 .
$$

\footnotetext{
${ }^{1}$ The coefficient $\lambda_{\chi}$ can be derived from the values of the thermal conduction coefficient $\kappa\left(m_{\chi} / m_{i}\right)$ tabulated in Ref. [12]. It holds: $\lambda_{\chi}=\left(1 / l_{\chi}\right)\left[\Sigma_{i}\left(1 / l_{\chi, i} \kappa\right)\right]^{-1}$ where $l_{\chi, i}$ is the WIMP mean free path relative to the nucleus $i$.
}

Gould and Raffelt [14], by means of a Monte Carlo integration of the Boltzmann collision equation, showed that the Spergel and Press approximation, Eq. (10), overestimates the energy transfer typically by a factor of a few. Moreover, by studying simplified stellar models, they showed that it is possible to approximate energy transfer in the nonlocal regime by a conductive treatment if one applies a global "luminosity suppression factor." We follow their approach by using as a general expression for the WIMP luminosity:

$$
L_{\chi}(r)=f(K) L_{\chi, \text { cond }}(r),
$$

where

$$
f(K)=\frac{1}{\left(K / K_{0}\right)^{2}+1},
$$

and $K_{0}=0.4$ is the value of the Knudsen parameter for which the WIMP energy transport is most efficient. We remark that other approaches are possible. In Refs. [15] and [29,30], for example, a suitable interpolation of Eqs. (7) and (9) is used as a general expression for WIMP luminosity. We have checked numerically that our results are essentially independent of the chosen approach.

As mentioned before, WIMPs can interact with nuclei either via coherent interactions or via spin-dependent ones. In the first case the WIMP cross section on the $i$ th nucleus of mass $m_{i}$ is related to the corresponding WIMP-nucleon cross section, $\sigma_{c}$, by

$$
\sigma_{i}=\sigma_{c}\left(\frac{m_{i}}{m_{n}}\right)^{4}\left(\frac{m_{\chi}+m_{n}}{m_{\chi}+m_{i}}\right)^{2}
$$

where $m_{n}$ is the nucleon mass. With this assumption, Eq. (6) may be rewritten as

$$
\frac{1}{l_{\chi}(r)}=\frac{1}{l_{n}(r)} \sum_{i} X_{i}(r)\left(\frac{m_{i}}{m_{n}}\right)^{3}\left(\frac{m_{\chi}+m_{n}}{m_{\chi}+m_{i}}\right)^{2},
$$

with

$$
l_{n}(r)=\frac{m_{n}}{\sigma_{c} \rho(r)} \simeq\left(\frac{1.6 \times 10^{-37} \mathrm{~cm}^{2}}{\sigma_{c}}\right)\left(\frac{150 \mathrm{~g} \mathrm{~cm}^{-3}}{\rho(r)}\right) \cdot R_{\odot} .
$$

In the Sun the abundances $X_{i}$ for nuclei heavier than hydrogen and helium are much smaller than those of $\mathrm{H}$ and $\mathrm{He}$. However, in the sum over elements of Eq. (15), for heavy nuclei (mainly for iron) the reduction due to $X_{i}$ is largely compensated by the coherence effect displayed by Eq. (14).

On the other hand, as far as spin-dependent interactions are concerned, the contribution of hydrogen is overwhelmingly dominant over the contributions of other nuclear species with nonvanishing spins. Therefore, from now on we identify $\sigma_{s d}$ with the spin-dependent WIMP-proton cross section.

In the following all WIMP matter interactions will be expressed in terms of $\sigma_{c}$ and/or $\sigma_{s d}$. The notation $\sigma_{p}$ will be used to denote a generic WIMP-proton cross section; $\sigma_{p}$ has 
to be identified with $\sigma_{c}$ (or $\sigma_{s d}$ ), whenever coherent (or spin dependent) interactions are considered.

\section{WIMPS AND THE CENTRAL SOLAR STRUCTURE}

The efficiency of WIMP energy transport depends on several parameters. Specifically, it depends on the total number $N_{\chi}$ of WIMPs in the Sun, on the WIMP mass $m_{\chi}$ and on the WIMP-nucleon cross section $\sigma_{p}$. In order to determine the region of the WIMP parameter space where the WIMP energy transport is most efficient it is useful to define the quantity

$$
\delta=\lim _{r \rightarrow 0} \frac{L_{\chi}(r)}{L(r)},
$$

where $L$ is the radiative luminosity of the Sun. It is easy to show that

$$
\delta=\left[\frac{1}{\left(K / K_{0}\right)^{2}+1}\right] \frac{\kappa_{\gamma}(0)}{\kappa_{\chi}(0)},
$$

where $\kappa_{\gamma, \chi}(0)$ are the opacities at the solar center for radiation and WIMPs.

In Figs. 1 and 2 we show the lines in the plane $\left(\sigma_{p}, N_{\chi}\right)$ which correspond to fixed values of $\delta$. We have chosen $m_{\chi}$ $=50 \mathrm{GeV}$ as a representative value for the WIMP mass. Figure 1 refers to coherent WIMP scattering, while Fig. 2 is obtained for spin-dependent WIMP interactions. The lines have been calculated by using the physical and chemical parameters of the Sun as they are obtained by a standard evolutionary code [33].

The qualitative behavior of the iso- $\delta$ lines is easily understood. The efficiency of the WIMP energy transport is proportional to $1 / \sigma_{p}$ (i.e., to $K$ ) in the conductive regime and to $\sigma_{p}$ (i.e., to $K^{-1}$ ) in the nonlocal regime. The transition between the two regimes occurs at $K=l_{\chi} / r_{\chi} \simeq 0.4$, i.e., at a cross section $\sigma_{t}$ which corresponds, for coherent scattering, to the value $\sigma_{t} \simeq 10^{-37} \mathrm{~cm}^{2}$ and, for spin-dependent interactions, to $\sigma_{t} \simeq 10^{-34} \mathrm{~cm}^{2}$. The difference in the values of the transition cross sections $\sigma_{t}$ is due to the fact that, for the spin-independent case, interactions on elements heavier than hydrogen are important, due to the coherence effect in the scattering which is manifest in Eq. (14), while for spindependent interactions no coherence is present and therefore only scattering on hydrogen (which is largely more abundant than other elements with nonvanishing spin) matters.

To be more quantitative, we can also show the analytic expression of the iso- $\delta$ lines as a function of $\sigma_{p}$ :

$$
N_{\chi}\left(\delta, \sigma_{p}\right)=N(\delta)\left[\left(\sigma_{p} / \sigma_{t}\right)+\left(\sigma_{p} / \sigma_{t}\right)^{-1}\right],
$$

where $N(\delta)$ is given by the condition

$$
\kappa_{\gamma}(0)=\delta \kappa_{\chi}\left(0 ; l_{\chi}=0.4 r_{\chi}\right)
$$

which gives

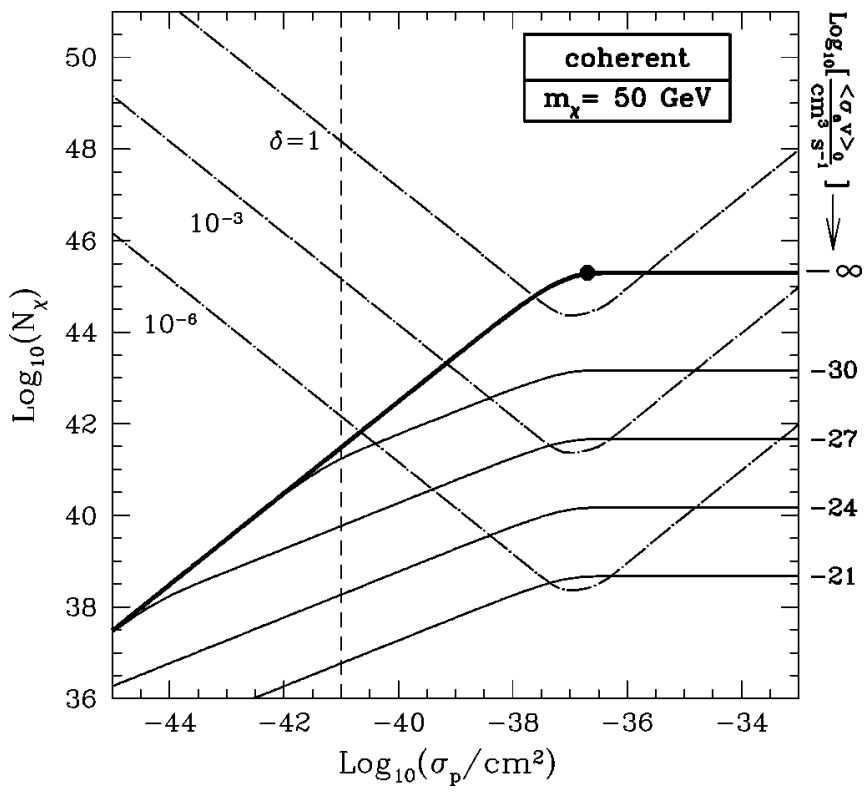

FIG. 1. The solid curves denote the total number $N_{\chi}$ of WIMPs accumulated in the Sun's core due to gravitational capture as a function of the WIMP-nucleon cross section, for a WIMP mass of $50 \mathrm{GeV}$ and for the case of purely coherent WIMP-nucleus interactions. The thick solid line refers to WIMPs which do not annihilate once captured. The thin solid lines refer to annihilating WIMPs, for increasing values of the zero-temperature thermally averaged WIMP-antiWIMP annihilation cross section: $\left\langle\sigma_{a} v\right\rangle_{0}=10^{-30}$, $10^{-27}, 10^{-24}, 10^{-21} \mathrm{~cm}^{3} \mathrm{~s}^{-1}$. The dot-dashed lines show the contours of constant values of the parameter $\delta$, defined in Eq. (17), which quantifies the efficiency of energy transport of WIMPs with respect to photons at the center of the Sun. The vertical dashed line denotes the upper limit on the WIMP-nucleon elastic cross section for coherent interactions. The large dot identifies the WIMP parameters for which the WIMP effect on the Sun's energetic is maximal.

$$
\frac{N(\delta)}{\pi^{3 / 2} r_{\chi}^{3}}=\delta \frac{4 a_{\mathrm{rad}} T_{c}^{3}}{3} \sqrt{\frac{m_{\chi}}{T_{c}}} \frac{l_{\gamma}}{0.4 r_{\chi} \lambda_{\chi}},
$$

and $l_{\gamma} \simeq 5 \times 10^{-3} \mathrm{~cm}$ is the photon mean free path at the center of the Sun.

We remark that, since WIMP radial distribution is rapidly decreasing [see Eq. (3)], the parameter $\delta$ essentially provides an upper limit for the ratio $L_{\chi} / L$ in the Sun. As a consequence, the condition that $\delta$ is not too small should be used as a necessary (but not sufficient) condition for WIMPs to have relevant effects on the Sun. This means the following.

(i) We can safely conclude that regions of the parameter space for which $\delta \leqq 10^{-2}$ are not interesting. This point can be easily understood. WIMPs modify the relation between the luminosity and the gradient of temperature in the Sun. This essentially corresponds to a redefinition of the Sun radiative opacity $\kappa_{\gamma}$. The effect is maximal at the center of the Sun where one has $\kappa_{\gamma} \rightarrow \kappa_{\gamma} /(1+\delta)$. The radiative opacity is affected by uncertainties at the level of a few percent [34]. This means that we cannot disentangle WIMP effects if $\delta$ is smaller than $10^{-2}$.

(ii) We cannot conclude, at this stage, that regions in which $\delta$ is large (say e.g. $\delta>1$ ) correspond to sizeable 


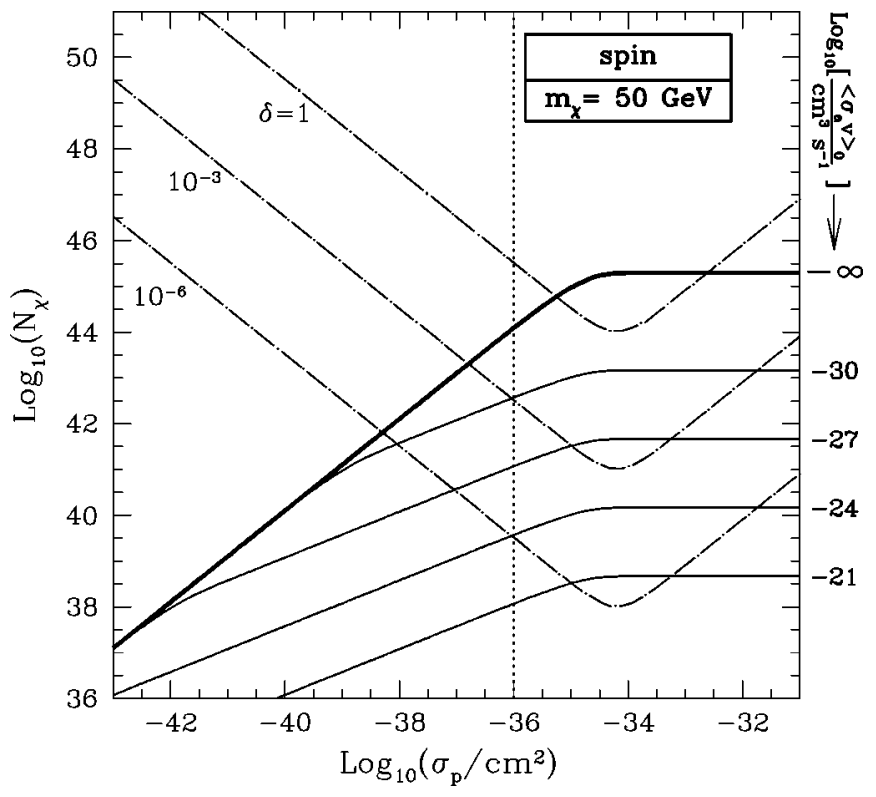

FIG. 2. The same as in Fig. 1, for spin-dependent WIMPnucleus interactions. The vertical dotted line denotes the upper limit on the WIMP-nucleon cross section for Z-boson mediated spindependent interactions.

modifications of the solar structure. The parameter $\delta$ is, in fact, a local parameter. A large $\delta$ only implies that WIMPs give a large contribution to the energy transport at the center of the Sun. In order to understand whether WIMPs have relevant effects on global properties of the Sun, we also have to compare the radius $r_{\chi}$ of the WIMPs extension region with the relevant length scales in the Sun (e.g., the dimension of the neutrino production region, the temperature height scale).

\section{THE NUMBER OF WIMPS IN THE SUN}

Up to this point we have treated $m_{\chi}, \sigma_{p}$ and $N_{\chi}$ as independent quantities. However, this is not the case, as noted long ago in Ref. [16]. $N_{\chi}$ depends on the capture rate of the halo WIMPs by the Sun during its lifetime; in turn, this capture rate is proportional to the product of the WIMP density in the halo $\rho_{\chi}$ times $\sigma_{p}$, i.e., to the product $\xi \sigma_{p}$. Moreover, captured WIMPs may annihilate with captured antiWIMPs. Therefore $N_{\chi}$ also depends on the quantity $\left\langle\sigma_{a} v\right\rangle_{0}$, where $\sigma_{a}$ is the WIMP-antiWIMP pair annihilation cross section and $v$ is the relative velocity of the annihilating pair, while brackets indicate the average over the WIMP velocity distribution in the Sun core. Explicitly, one has [31]

$$
N_{\chi}=\Gamma_{c} \tau \tanh \left(t_{\odot} / \tau\right),
$$

where $t_{\odot} \simeq 4.5 \mathrm{Gyr}$ is the age of the Sun, $\Gamma_{c}$ is the WIMP capture rate and $\tau=1 / \sqrt{\Gamma_{c} C_{a}}$ is a time scale parameter which determines whether and when equilibrium between capture and annihilation occurs. The quantity $C_{a}$ is given by

$$
C_{a}=\frac{\left\langle\sigma_{a} v\right\rangle_{0}}{V_{0}}\left(\frac{m_{\chi}}{20 \mathrm{GeV}}\right)^{3 / 2}
$$

TABLE I. Sun composition, average mass fractions of the chemical elements in the Sun as obtained from Ref. [44] and values of the average reduced gravitational potential $\langle\phi\rangle_{i}$.

\begin{tabular}{lll}
\hline \hline Element & \multicolumn{1}{c}{$X_{i}$} & $\langle\phi\rangle_{i}$ \\
\hline $\mathrm{H}$ & 0.71 & 3.16 \\
$\mathrm{He}$ & 0.27 & 3.40 \\
$\mathrm{C}$ & $0.30 \times 10^{-2}$ & 3.23 \\
$\mathrm{~N}$ & $0.93 \times 10^{-3}$ & 3.23 \\
$\mathrm{O}$ & $0.84 \times 10^{-2}$ & 3.23 \\
$\mathrm{Ne}$ & $0.17 \times 10^{-2}$ & 3.23 \\
$\mathrm{Na}$ & $0.35 \times 10^{-4}$ & 3.23 \\
$\mathrm{Mg}$ & $0.65 \times 10^{-3}$ & 3.23 \\
$\mathrm{Al}$ & $0.56 \times 10^{-4}$ & 3.23 \\
$\mathrm{Si}$ & $0.70 \times 10^{-3}$ & 3.23 \\
$\mathrm{P}$ & $0.62 \times 10^{-5}$ & 3.23 \\
$\mathrm{~S}$ & $0.37 \times 10^{-3}$ & 3.23 \\
$\mathrm{Cl}$ & $0.78 \times 10^{-5}$ & 3.23 \\
$\mathrm{Ar}$ & $0.94 \times 10^{-4}$ & 3.23 \\
$\mathrm{Ca}$ & $0.65 \times 10^{-4}$ & 3.23 \\
$\mathrm{Ti}$ & $0.36 \times 10^{-5}$ & 3.23 \\
$\mathrm{Cr}$ & $0.17 \times 10^{-4}$ & 3.23 \\
$\mathrm{Mn}$ & $0.96 \times 10^{-5}$ & 3.23 \\
$\mathrm{Fe}$ & $0.12 \times 10^{-2}$ & 3.23 \\
$\mathrm{Ni}$ & $0.74 \times 10^{-4}$ & 3.23 \\
\hline \hline
\end{tabular}

with $V_{0} \simeq 2.3 \times 10^{28} \mathrm{~cm}^{3}$ for the Sun. In the limit in which $\left\langle\sigma_{a} v\right\rangle_{0} \rightarrow 0$, one has $\tau \rightarrow \infty$ and Eq. (22) becomes

$$
N_{\chi}=\Gamma_{c} t_{\odot} \equiv N_{\chi}^{\max } .
$$

$N_{\chi}^{\max }$ represents the upper limit for the WIMP number in the Sun at the present time.

The expression of the capture rate $\Gamma_{c}$ is rather involved. For a Maxwellian WIMP velocity distribution in the galactic halo it may be cast in the form [35]

$$
\Gamma_{c}=\sum_{i}\left(\frac{8}{3 \pi}\right)^{1 / 2}\left[\sigma_{i} \frac{\rho_{\chi}}{m_{\chi}} \bar{v}\right]\left[\frac{M_{i}}{m_{i}}\right]\left[\frac{3 v_{e s c}^{2}}{2 \bar{v}^{2}}\langle\phi\rangle_{i}\right] \xi(\infty) S_{i},
$$

where the cross sections $\sigma_{i}$ are related to $\sigma_{p}$ as discussed in the previous section, $M_{i}$ is the total mass of the $i$ th nucleus in the Sun, $v_{\text {esc }} \simeq 618 \mathrm{~km} \mathrm{sec}^{-1}$ is the escape velocity at the Sun's surface and $\bar{v}=270 \mathrm{~km} \mathrm{sec}^{-1}$ is the WIMP velocity dispersion. The quantities $\langle\phi\rangle_{i}$ denote the reduced gravitational potential $\phi(r)=v_{e s c}^{2}(r) / v_{e s c}^{2}$ averaged over the mass distribution of the $i$ th nucleus, as defined in Eq. (A2) in the Appendix. The quantity $\xi(\infty) \simeq 0.75$ is a suppression factor due to the motion of the Sun through the halo and the factor $S_{i}$ includes the effects of WIMP-nucleus mass mismatch and finite target dimensions (note that the $\sigma_{i}$ are point-like cross sections). The expression of $S_{i}$ is given in the Appendix for completeness. The chemical composition of the Sun and the values of the quantities $\langle\phi\rangle_{i}$, which have been used in our calculations, are given in Table I. We note that for relatively heavy WIMPs like the ones which are considered in this 
analysis $\left(m_{\chi} \geq 30 \mathrm{GeV}\right)$ the capture in the Sun for spinindependent interaction is not dominated by scattering on hydrogen, even though this is the most abundant element, but instead by interactions on heavier elements. For instance, for a $50 \mathrm{GeV}$ WIMP, capture is dominated by $\mathrm{He}, \mathrm{O}$ and Fe. The smaller abundance of heavier elements is largely compensated by the coherence effect in the cross section shown in Eq. (14). On the contrary, in the case of spin-dependent interactions the only relevant capture process is on hydrogen, since in this case no coherence effect is present and therefore capture is dominated by interactions with the most abundant element which possesses spin, i.e., hydrogen.

Equation (25) holds when the cross section $\sigma_{p}$ is small enough so that multiple scatterings can be neglected. However, when $\sigma_{p}$ is so large that every WIMP crossing the Sun is captured, $\Gamma_{c}$ saturates to a maximal value, and Eq. (25) is replaced by

$$
\Gamma_{c, s a t}=\left(\frac{8}{3 \pi}\right)^{1 / 2}\left[\frac{\rho_{\chi}}{m_{\chi}} \bar{v}\right]\left[\frac{M_{i}}{m_{i}}\right]\left[\zeta+\frac{3 v_{e s c}^{2}}{2 \bar{v}^{2}}\right] \xi(\infty) \pi R_{\odot}^{2},
$$

where $\zeta \simeq 1.77$. In Eq. (26), the first term in the last square brackets refers to WIMPs whose orbit crosses the Sun even without gravitational deflection, while the second term is a gravitational focusing factor and corresponds to those WIMPs whose orbits pass through the Sun because of gravitational deflection. One can easily see that for the Sun the focusing factor is dominant over the purely "geometrical" one, since the velocity dispersion of WIMPs in the Galaxy is a factor of 2 smaller than the escape velocity at the Sun's surface.

The behavior of the capture rate, and consequently of the total number $N_{\chi}$ of WIMPs captured by the Sun, as a function of the WIMP parameters is now easily understood. At fixed $m_{\chi}, N_{\chi}$ increases linearly with $\sigma_{p}$ up to the saturation level. For $m_{\chi}=50 \mathrm{GeV}$, this behavior can be seen in Fig. 1 (coherent interactions) and Fig. 2 (spin-dependent interactions), where the thick solid line represents $N_{\chi}$ as a function of $\sigma_{p}$ for the case of no-annihilation $\left(\sigma_{a}=0\right)$ and the thin solid lines show $N_{\chi}$ for some representative nonvanishing values of $\left\langle\sigma_{a} v\right\rangle_{0}$. In calculating $N_{\chi}$ as a function of $\sigma_{p}$, we have set $\rho_{\chi}=\rho_{l}$, i.e., $\xi=1$. For $\rho_{l}$ we have used, here and throughout the paper, the default value $\rho_{l}=0.3 \mathrm{GeV} \mathrm{cm}^{-3}$. The current uncertainty on $\rho_{l} \quad\left(0.2 \mathrm{GeV} \mathrm{cm}^{-3} \lesssim \rho_{l}\right.$ $\lesssim 0.7 \mathrm{GeV} \mathrm{cm}^{-3}$, for an isothermal galactic halo [7]) may actually increase or reduce $N_{\chi}$ by at most a factor of 2 . The no-annihilation line represents, for a given WIMP mass, the maximal number of WIMPs that can be captured by the Sun during its lifetime. If annihilation is present, $N_{\chi}$ is obviously smaller, depending on the strength of the annihilation cross section. Results very similar to those shown in Figs. 1 and 2 are obtained for different WIMP masses. Notice that the transition from the linear regime to the saturation level occurs at quite different values for the relevant WIMP-nuclen cross section in the two limiting cases of pure coherent and pure spin-dependent interactions. This is again due to the fact that for WIMPs heavier than tens of $\mathrm{GeV}$, scattering on heavy nuclei in the Sun is enhanced for coherent interactions, and this shifts the transition from the linear to the saturation regime toward WIMP-nucleon cross sections smaller than in the spin-dependent case.

The previous discussion may be easily extended to the case in which the WIMP under consideration does not provide the total amount of local dark matter (i.e., $\xi<1$ ). In this case, the relevant quantities are conveniently plotted in the plane $\xi \sigma_{p}-N_{\chi}$. For the case of coherent interactions the results are displayed in Fig. 3, whose four panels refer to different representative values: $\xi=1,0.1,0.01,0.001$. In each panel the dashed curve denotes a representative iso- $\delta$ line with $\delta=1$ and the solid line displays $N_{\chi}$ as a function of $\xi \sigma_{p}$ for the limiting case $\sigma_{a}=0$. For the sake of comparison, the top-left panel repeats the case already shown in Fig. 1 ( $\xi$ $=1$ ). The features of the panels with $\xi<1$ are easily understood in terms of those represented in the top-left panel: (i) the iso- $\delta$ curve simply shifts horizontally to the left by an amount equal to $\xi$, since $\delta$ depends on the scattering cross section $\sigma_{p}$ but it does not depend on $\xi$; (ii) the slanted part of the solid line is unchanged since $\Gamma_{c}$ in the linear regime, as given by Eq. (25), is a function of the product $\xi \sigma_{p}$; (iii) the flat part of the solid line (saturation regime) is lowered by an amount $\xi$ since $\Gamma_{c, s a t}$ in Eq. (26) depends on $\xi$ but is independent of $\sigma_{p}$. We explicitly consider the possibility of subdominant WIMPs since this is a feature which can naturally occur for specific dark matter candidates, like, for instance, the neutralino, as it will be discussed in Sec. VII.

We are now able to understand which regions of WIMP parameter space potentially lead to observable modification of the solar structure, by comparing the iso- $\delta$ lines with the curves which describe the number of WIMPs in the Sun. The first result is that the WIMP maximum effect occurs when annihilation is negligible and for scattering cross sections in the interval $10^{-38} \mathrm{~cm}^{2} \lesssim \sigma_{c} \lesssim 10^{-36} \mathrm{~cm}^{2}$ for coherent interactions and $10^{-35} \mathrm{~cm}^{2} \lesssim \sigma_{s d} \lesssim 10^{-33} \mathrm{~cm}^{2}$ for spin-dependent interactions. These cross sections correspond to a situation in which the number of WIMPs in the Sun has essentially reached its saturation value and the WIMP energy transport is maximally efficient (i.e., $K \sim K_{0}$ ). This is manifest in Figs. 1 and 2 for $m_{\chi}=50 \mathrm{GeV}$ and occurs also for all the WIMP mass range $m_{\chi} \geq 30 \mathrm{GeV}$ under study in the present paper. The value of $\delta$ which corresponds to this maximal effect is $\delta \sim 10$, both for coherent and spin-dependent interactions. We will use this information in the next sections where we will calculate the maximal deviation of the Sun properties when WIMPs are incorporated in its interior.

We stress that, for the WIMP mass range considered in the present paper, the process of WIMP capture cannot produce modifications of the Sun which refer to values of $\delta$ larger than about 10, for any value of the scattering cross section. Independent limits on the WIMP-nucleon interactions, like the ones that are obtained from direct search experiments, can further bound the values of $\delta$ that can be reached. This is the case for coherent interactions, for which direct search experiments have already been able to set stringent bounds. For $m_{\chi}=50 \mathrm{GeV}$, the upper limit on $\xi \sigma_{c}$ is shown in Fig. 1 by a vertical dashed line. From this figure, we can therefore see that once the direct detection constraint is taken into 

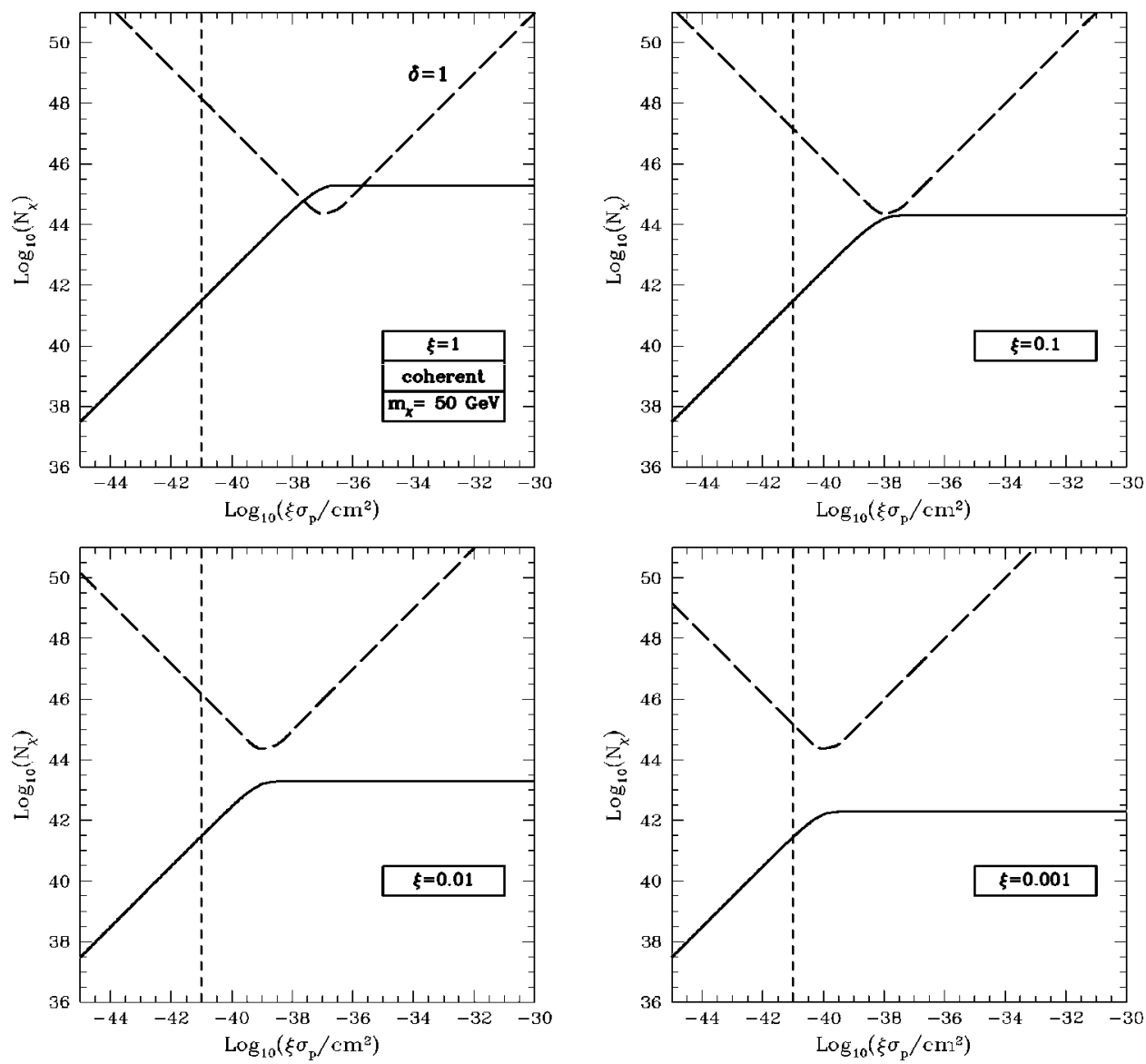

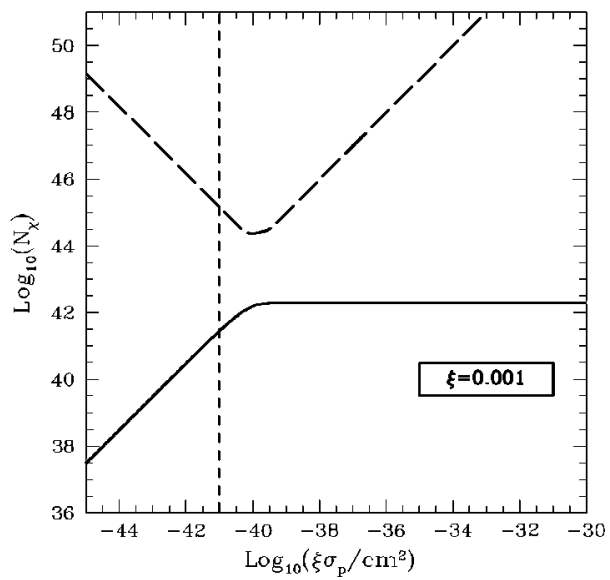

FIG. 3. The same as in Fig. 1, plotted as a function of $\xi \sigma_{p}$, for different values of the rescaling parameter $\xi$. The solid lines denote the total number of WIMPs accumulated in the Sun for a nonannihilating WIMP. The dashed lines show the contours of iso- $\delta$ for $\delta=1$. The vertical dashed lines indicate the upper limit on $\xi \sigma_{p}$ for coherent interactions. account, the largest values of $\delta$ which can be obtained are of the order of $10^{-6}-10^{-7}$, which are far too small to lead to observable effects, as already discussed at the end of Sec. III. Once rescaling is considered, the behaviors of the iso- $\delta$ and $N_{\chi}^{\max }$ lines plotted in Fig. 3 show that $\delta$ 's of the order of $10^{-3}-10^{-2}$ can be reached. These values are close to the necessary (but not sufficient) condition for WIMPs to have observable effects on the Sun (i.e. $\delta \gtrsim 10^{-2}$ ), introduced in Sec. III, and therefore they need to be analyzed in detail.

In the case of spin-dependent interactions, direct search experiments are also setting bounds on $\xi \sigma_{s d}$, but these limits are not model independent since they rely on the way the interaction of WIMPs with the spin of the nucleus is realized [5]. For the representative case of a $Z$-boson mediated interaction and for $m_{\chi}=50 \mathrm{GeV}$, the present upper limit is shown in Fig. 2 by a dotted vertical line. Since this line cannot be assumed to be a model independent bound, in the case of spin-dependent interactions we are left with the possibility to reach values of $\delta \sim 10$.

Up to this point we have quantified the amount of possible deviations induced by a generic WIMP in terms of the parameter $\delta$. We now turn to discuss the possibility to experimentally observe such modifications by means of helioseismology and of the boron solar neutrino flux.

\section{WIMPS AND HELIOSEISMOLOGY}

Helioseismology has provided very accurate information on the solar structure and it has been able to establish severe constraints and tests of the solar standard model (SSM) calculations. For instance, helioseismology accurately determines the depth of the convective envelope $R_{b}$ and the photospheric helium abundance $Y_{p h}$. Moreover, by inversion of helioseismic data, one can determine the (squared isothermal) sound speed in the solar interior, $u=P / \rho$, with high accuracy ( $P$ and $\rho$ denote the pressure and density inside the Sun). In Fig. 4, we show the uncertainty in the helioseismic determination of $u$ as a function of the radial coordinate $R / R_{\odot}$. The light band corresponds to the so-called " $3 \sigma$ " errors which have been estimated conservatively by adding linearly all known individual uncertainties [18]. If uncertainties are added in quadrature, the global error is about one third. This yields the so called " $1 \sigma$ " errors which are shown by the dark band in Fig. 4. This latter procedure was also used by Bahcall et al. [36] with similar results. For intermediate values of the radial coordinate, the uncertainty in the squared sound speed is at the level of $10^{-3}$, while in the innermost part of the Sun, as well as on the surface, it is at the percent level. We remark that, as found in Refs. [18] and [36], uncertainties corresponding to statistical errors of the frequency measurements are generally much smaller than the "systematical uncertainties" of the inversion method. These latter include the choice of the reference model and of the free parameters in the inversion procedure. These uncertainties are particularly important close to the solar center, since there are very few $p$-modes that sample this region well. The errors quoted above include both statistical and systematic uncertainties. On the other hand, Refs. [37] and [38] present 


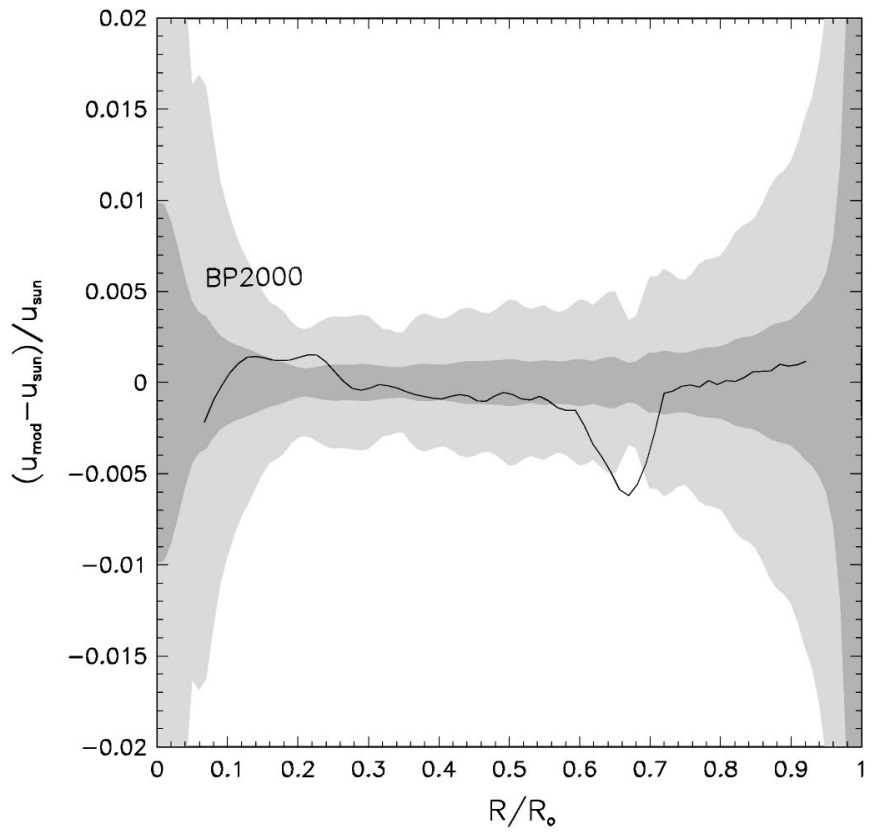

FIG. 4. Uncertainty bands in the reconstruction of the squared isothermal sound speed $u$ in the Sun interior. The light region corresponds to the conventional " $3 \sigma$ " error band, while the dark band identifies the conventional " $1 \sigma$ " range. The solid line corresponds to the fractional difference between $u$ determined from observational data and $u$ calculated from the BP2000 solar model [39].

sound speed profiles with errors which look significantly smaller than our estimate. This occurs since "the quoted uncertainties include only the contribution arising from the frequency observations," as clearly stated in [38].

Figure 4 also shows the difference between $u$ as predicted by the SSM of Ref. [39] and the helioseismological determination $u_{\text {sun }}$, normalized to $u_{\text {sun }}$. We notice that the SSM accurately reproduces $u$ for all the radial profile.

In order to understand whether helioseismology can tell us something about WIMPs, we have constructed solar models for values of the WIMP parameters which maximize the role of WIMPs in the energetics of the Sun, irrespective of possible bounds derivable from direct search experiments. As discussed in the previous section, this corresponds to $\delta$ $\sim 10$. For $m_{\chi}=50 \mathrm{GeV}$, this is shown, for instance, by the large dot on the "no-annihilation line" in Fig. 1. We have taken into account the time evolution of the number of WIMPs in the Sun and we have described WIMP energy transport at each stage of Sun evolution according to Eq. (12). The sound speed profiles obtained for the representative WIMP masses $m_{\chi}=30,50$ and $100 \mathrm{GeV}$ are compared with the sound speed profile of the SSM in Fig. 5. Larger WIMP masses lead to even smaller effects. For any WIMP mass, smaller values of $\delta$ clearly correspond to smaller effects.

The first observation is that, even in the most favorable situation, WIMPs produce a modification of the sound speed profile which is much smaller than the accuracy of present helioseismic determinations. The smallness of the effect is explained by two main reasons. First, WIMPs with masses larger than tens of $\mathrm{GeV}$ are confined in a small region of the Sun of radius $R \lesssim 0.02 R_{\odot}$. In this region the relevant physi-

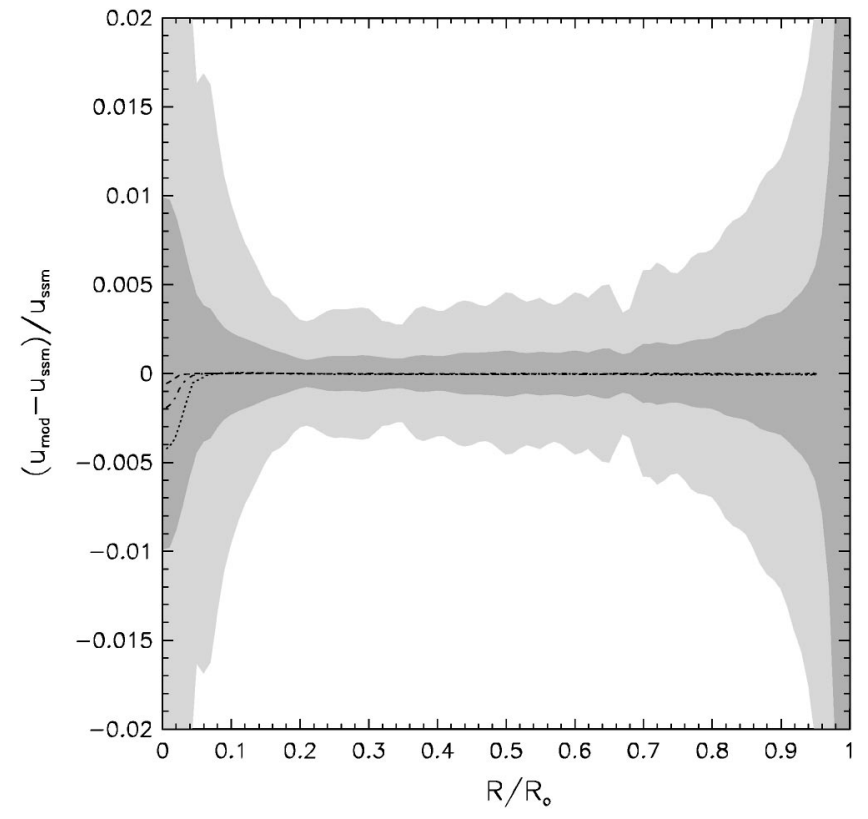

FIG. 5. Relative difference of the squared isothermal sound speed in the Sun interior $u$ calculated for a standard solar model and for solar models with accreting WIMPs, as a function of the radial coordinate $R / R_{\odot}$. The light region corresponds to the conventional " $3 \sigma$ " error band, while the dark band identifies the conventional " $1 \sigma$ " range. The dotted, dot-dashed and dashed lines refer to solar models with accreting WIMPs of masses $m_{\chi}=30,50,100 \mathrm{GeV}$ and coherent scattering cross sections which, for each mass, maximize the effect of the presence of WIMPs in the Sun.

cal quantities (temperature, luminosity, etc.) have small variations. As a consequence, even if WIMPs affect sizeably the gradient of these quantities (e.g. the temperature gradient), this translates into small modifications of their "global" radial profile. Second, a compensation occurs between two different effects. This can be easily understood in terms of the fact that, from the perfect gas law, which describes to a good approximation most of the solar core, one has $u$ $=P / \rho \simeq T / \mu$, where $\mu$ is the mean molecular weight. WIMPs produce a quasi-isothermal core, thus decreasing the central temperature $T_{c}$ of the Sun. At the same time, however, with a lower $T_{c}$ one has smaller nuclear reaction rates. This translates into a slower helium production at the center of the Sun and, thus, into a decrease of the mean molecular weight. When considering $u \simeq T / \mu$, the effects of the variations of $T$ and $\mu$ compensate, leaving the sound speed profile almost unaffected.

The results shown in Fig. 5 refer to a situation which maximizes the effect of the presence of WIMPs in the Sun: $\delta \sim 10$. As discussed in the previous section, for spindependent interactions, this value of $\delta$ can be reached, while for coherent interaction $\delta$ 's of the order of at most $10^{-3}-10^{-2}$ can be obtained. Our results show that in neither case (spin-depenent nor coherent) can the presence of WIMPs in the Sun affect $u$ to a level which is currently accessible by helioseismology.

We notice also that the necessary (but not sufficient) condition stated in Sec. III, i.e., that $\delta$ must be larger than about 
TABLE II. Fractional variations of the central temperature of the Sun $T_{c}$, of the squared isothermal sound speed in the Sun interior and of the ${ }^{8} \mathrm{~B}$ neutrino flux, calculated in solar models where WIMPs are accreted in the Solar core. For each model, the WIMP-nucleon scalar cross section has been set at the value which maximizes the effect of the presence of WIMPs in the Sun.

\begin{tabular}{cccc}
\hline \hline$m_{\chi}(\mathrm{GeV})$ & $\delta T_{c} / T_{c}$ & $\delta u_{c} / u_{c}$ & $\delta \Phi_{B} / \Phi_{B}$ \\
\hline 30 & $-2.1 \times 10^{-2}$ & $-4 \times 10^{-3}$ & $-4.5 \times 10^{-2}$ \\
50 & $-1.2 \times 10^{-2}$ & $-2 \times 10^{-3}$ & $-1.3 \times 10^{-2}$ \\
100 & $-5.2 \times 10^{-3}$ & $-6 \times 10^{-4}$ & $-2.1 \times 10^{-3}$ \\
\hline \hline
\end{tabular}

$10^{-2}$ in order to possibly have an observable effect, may be reinforced: in fact our detailed calculation shows that, for WIMP masses above $30 \mathrm{GeV}$, not even $\delta \sim 10$ is sufficient to produce a currently observable effect.

In summary, the results of this section show that no significant information about WIMPs with masses $m_{\chi}$ $\gtrsim 30 \mathrm{GeV}$ can be obtained at present from helioseismic data, for any value of the WIMP-nucleon cross section.

Conclusions conflicting with ours were derived in Ref. [29]. This may be traced back to the fact that in Ref. [29] the accuracy of the squared sound speed is taken to be of about $0.1 \%$ also in the innermost part of the Sun core relevant for WIMP confinement, say $R \leq 0.02 R \odot$. We argued above that, at these small radii, the accuracy of the helioseismic determination is significantly worse.

\section{WIMPS AND THE BORON NEUTRINO FLUX}

From the previous discussion, one could expect that the Sun central temperature $T_{c}$ is more sensitive than the central sound speed to the presence of WIMPs in the Sun. This is actually the case, as can be seen in Table II, which shows that WIMPs produce variations of $T_{c}$ at the percent level, while the central sound speed $u_{c}$ is affected at most at the level of $10^{-3}$.

Unfortunately, the central temperature of the Sun is not directly observable. The most direct information on it comes from the measurements of the solar neutrino fluxes. In particular, the comparison between the SNO charged current and super-Kamiokande data [21-26] has allowed to determine, in a model independent way, the ${ }^{8} \mathrm{~B}$ neutrino flux produced in the Sun with an accuracy of about $20 \%$. $^{2}$ This result, under the assumption that the ${ }^{8} \mathrm{~B}$ neutrino flux scales with the Sun central temperature as $\Phi_{\mathrm{B}} \propto T_{c}^{20}$, can be used to determine $T_{c}$ at the $1 \%$ level [28].

However, the presence of WIMPs in the core of the Sun

\footnotetext{
${ }^{2}$ The SNO experiment has recently reported the observations of neutral current $\nu$ interactions on deuterium [27] confirming the ${ }^{8} \mathrm{~B}$ neutrino flux predicted by SSM with an accuracy of about $12 \%$. This result is obtained under the assumption that the solar neutrino energy spectrum is undistorted. For this reason, in our analysis, we refer to the result obtained by comparing SNO charged current and super-Kamiokande data, which instead do not rely on this assumption, see Refs. [21,40].
}

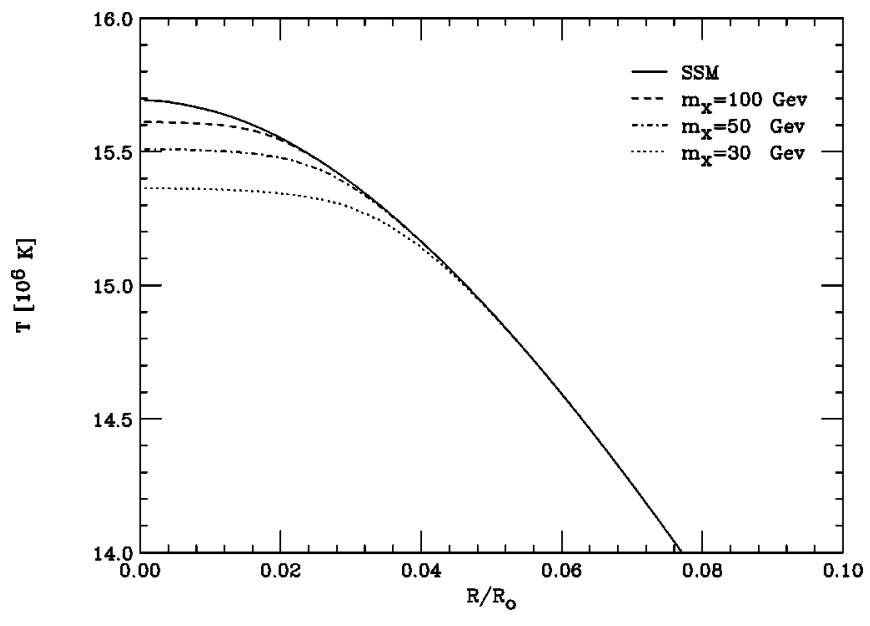

FIG. 6. Temperature profile of the standard solar model and of models of the Sun where WIMPs with masses of 30, 50 and 100 $\mathrm{GeV}$ are present. The coherent WIMP-nucleus scattering cross sections are chosen, for each mass, in order to maximize the effect of the presence of WIMPs in the Sun.

produces a very peculiar modification of the temperature profile for which the scaling law $\Phi_{\mathrm{B}} \propto T_{c}^{20}$ is not valid. WIMPs can sizeably affect the temperature profile only in the innermost part of the Sun, and in particular in a region which is smaller than the boron neutrino production region $\left(r_{B} \simeq 0.05 R_{\odot}\right)$. Figure 6 shows the radial profile of the Sun temperature in the SSM and in models of the Sun where WIMPs with masses of 30,50 and $100 \mathrm{GeV}$ are present. Again, we have chosen the WIMP parameters which maximize the effect of their presence in the Sun. Figure 6 clearly shows that even though the central temperature of the Sun can be lowered by the presence of WIMPs at the percent level (as it is reported also in Table II), nevertheless the temperature at $r_{B} \simeq 0.05 R_{\odot}$, where the boron neutrino production is maximal, is left practically unchanged.

In order to perform a quantitative analysis, one has therefore to look directly at the boron neutrino flux and not simply at the central temperature $T_{c}$. If this is done, it turns out that the solar models of Fig. 6, which correspond to WIMP masses equal to 30,50 and $100 \mathrm{GeV}$, predict variations of the boron neutrino flux equal to $\delta \Phi_{\mathrm{B}} / \Phi_{\mathrm{B}} \simeq-3.6 \%,-1.0 \%$ and $-0.1 \%$, respectively. These variations are well below the accuracy of the present determination of the boron neutrino flux. We remind the reader that, for each WIMP mass, we have optimized the other WIMP parameters in order to maximize the effect. Larger masses and WIMP annihilation would lead to even smaller deviations.

In conclusion, our results allow us to conclude that no information about WIMPs with massess $m_{\chi} \gtrsim 30 \mathrm{GeV}$ can be obtained from our present knowledge of the boron neutrino flux.

A previous independent analysis [30] concluded that WIMPs lighter than $60 \mathrm{GeV}$ are in conflict with the present accuracy in the determination of the boron neutrino flux, based on the fact that the presence of WIMPs in the core of the Sun can modify the central temperature $T_{c}$ at the $1 \%$ level. We should stress that this lower limit on the WIMP 


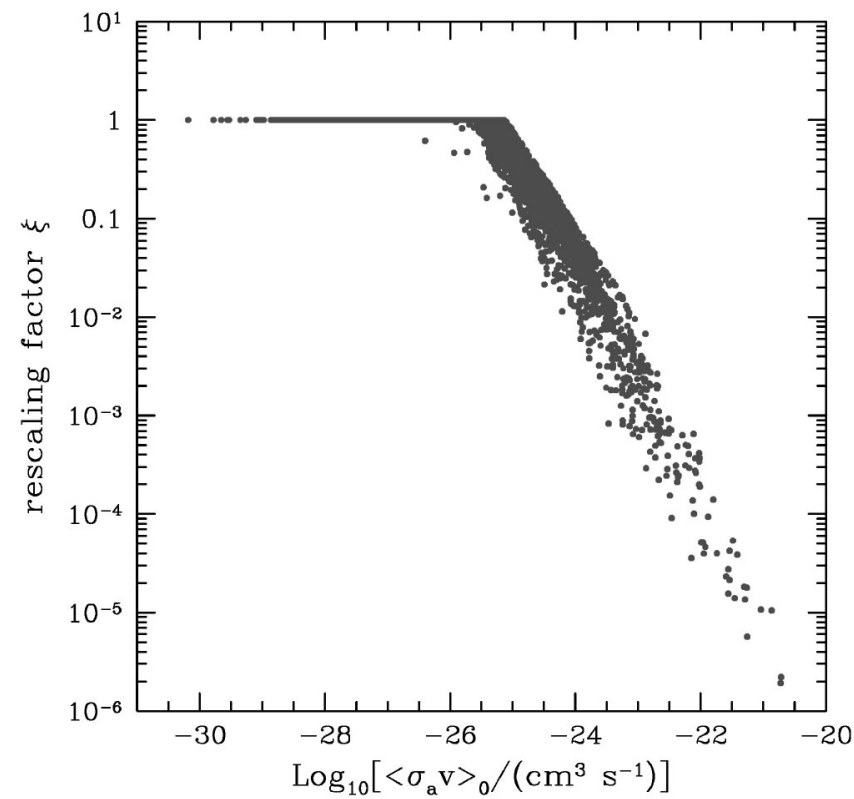

FIG. 7. Rescaling parameter $\xi=\min \left(1, \Omega_{\chi} h^{2} / 0.05\right)$ as a function of the zero-temperature thermally averaged neutralino selfannihilation cross section $\left\langle\sigma_{a} v\right\rangle_{0}$ in the effective minimal supersymmetric standard model (MSSM).

mass does not apply, since, as we have discussed above, WIMPs, though capable of changing $T_{c}$ at the percent level, nevertheless are too concentrated in the interior of the Sun to produce sizeable modifications of the boron neutrino flux, which is the quantity experimentally measured.

\section{ONE REALIZATION OF WIMP: THE NEUTRALINO}

It is instructive to discuss how some of the previous properties are realized in the case of one of the favorite WIMP candidates: the neutralino. In Figs. 7-9 we show some of the relevant properties.

The supersymmetric scheme we are adopting here is an effective minimal supersymmetric extension of the standard model (MSSM). Its parameters are defined directly at the electroweak scale and represent the minimum set necessary to shape the essentials of the theoretical structure of the model and of its particle content. We refer to Ref. [41] for details on the theoretical aspects and on the updated experimental bounds.

The neutralino-nucleon cross sections, both coherent and spin-dependent, are calculated in terms of the couplings and supersymmetric particle masses as discussed, for instance, in Refs. $[5,10]$. The parameter $\xi$ is evaluated by using a standard rescaling procedure [42] which relates the fraction of local neutralino dark matter in the Galaxy to its fractional abundance in the Universe, i.e., to its relic abundance $\Omega_{\chi} h^{2}$ :

$$
\xi=\min \left[1, \frac{\Omega_{\chi} h^{2}}{\left(\Omega_{m} h^{2}\right)_{\min }}\right],
$$

where $\left(\Omega_{m} h^{2}\right)_{\min }$ is the minimum value of $\Omega_{m} h^{2}$ compatible

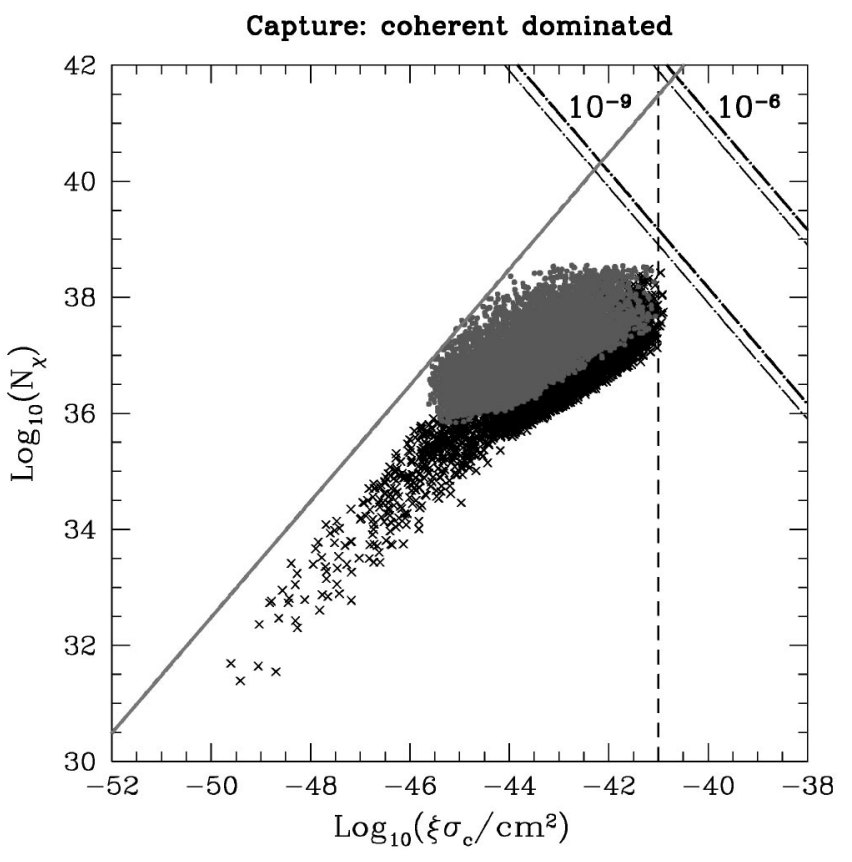

FIG. 8. Total number $N_{\chi}$ of neutralinos accumulated in the Sun as a function of $\xi \sigma_{c}$ in the effective MSSM. Only configurations where the total capture rate is dominated by coherent interactions are displayed. Neutralino masses are in the range $50 \mathrm{GeV}<m_{\chi}$ $<500 \mathrm{GeV}$. Dots denote configurations where the neutralino is the dominant dark matter component: $0.05<\Omega_{\chi} h^{2}<0.3$ (i.e., $\xi=1$ ). Crosses refer to configurations where the neutralino is a subdominant dark matter component: $\Omega_{\chi} h^{2}<0.05$ (i.e., $\xi<1$ ). The slanted solid line shows $N_{\chi}$ for $m_{\chi}=50 \mathrm{GeV}$ and for the limiting case of nonannihilating WIMPs. The dot-dashed lines denote iso- $\delta$ contours. For each pair, the upper line refers to the iso- $\delta$ curve for $m_{\chi}=50 \mathrm{GeV}$ and $\xi=1$, while the lower line refers to the same iso$\delta$ and $\xi$, for $m_{\chi}=500 \mathrm{GeV}$. The vertical dashed lines indicate the upper limit on $\xi \sigma_{p}$ for coherent interactions.

with halo properties. Here we set $\left(\Omega_{m} h^{2}\right)_{\min }=0.05$. The present-day neutralino relic abundance has been calculated according to Ref. [43].

To establish some relevant order of magnitude for the annihilation cross section $\sigma_{a}$ it is useful to recall the approximate expression $\Omega_{\chi} h^{2} \sim 3 \times 10^{-27} \mathrm{~cm}^{3} \mathrm{~s}^{-1} /\left\langle\sigma_{a} v\right\rangle_{\text {int }}$, where $\left\langle\sigma_{a} v\right\rangle_{\text {int }}$ denotes the thermal average of the product $\left(\sigma_{a} \cdot v\right)$ integrated from the freeze-out temperature $T_{f}$ to the present-day one. For instance the upper bound $\Omega_{\chi} h^{2} \leqq 0.3$ translates into a lower bound $\left\langle\sigma_{a} v\right\rangle_{\text {int }} \gtrsim 10^{-26} \mathrm{~cm}^{3} \mathrm{sec}^{-1}$.

In Fig. 7 we display the correlation between $\xi$ and $\left\langle\sigma_{a} v\right\rangle_{0}$, calculated by varying the MSSM parameters and applying to the model the relevant experimental constraints. Figure 7 shows that for a neutralino in the MSSM, the rescaling factor is always smaller than 1 for values of $\left\langle\sigma_{a} v\right\rangle_{0}$ larger than about $10^{-27}-10^{-26} \mathrm{~cm}^{3} \mathrm{~s}^{-1}$. Notice that $\left\langle\sigma_{a} v\right\rangle_{0}$ denotes the thermal average of the product $\left(\sigma_{a} \cdot v\right)$ in the Sun's core, and does not coincide with the relevant thermal average which enters in the calculation of the relic abundance.

We turn now to the graphic representations in the plane $\xi \sigma_{p}-N_{\chi}$. Figure 8 (Fig. 9) shows the scatter plot of the MSSM configurations for which the total capture rate is 


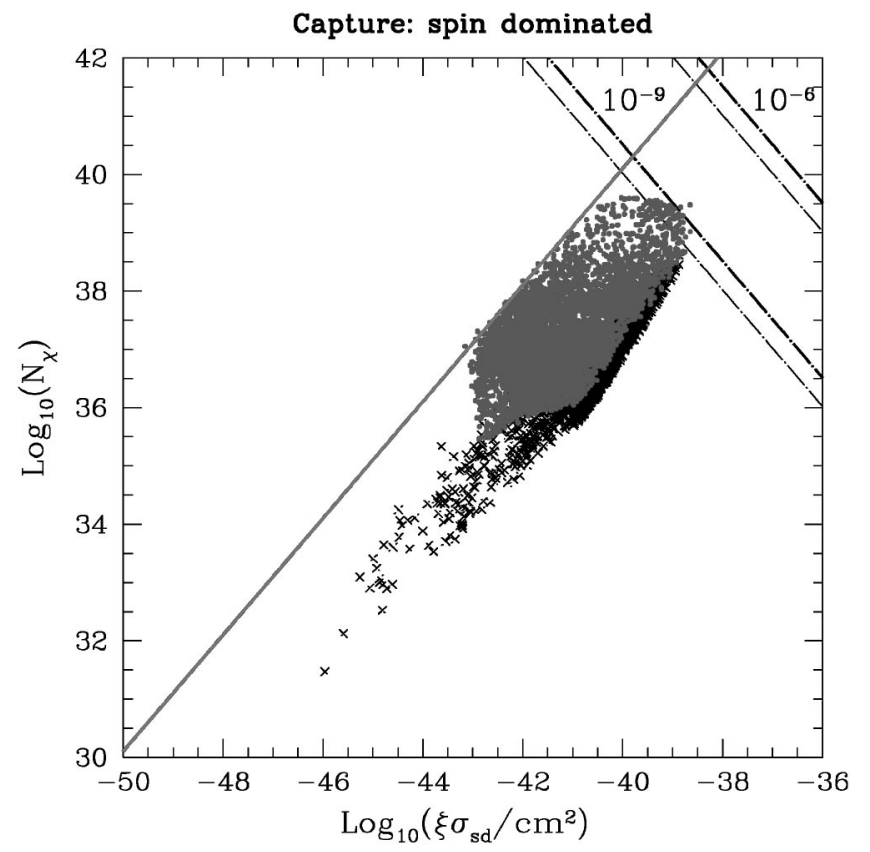

FIG. 9. The same as in Fig. 8, for MSSM configurations where the total capture rate is dominated by spin-dependent interactions.

dominated by coherent (spin-dependent) interactions and for the neutralino mass range: $50 \mathrm{GeV}<m_{\chi}<500 \mathrm{GeV}$, the lower bound arising from experimental constraints on the MSSM. The points are labeled according to the values of the neutralino relic abundance: dots denote configurations where the neutralino is the dominant dark matter component: 0.05 $<\Omega_{\chi} h^{2}<0.3$, i.e., $\xi=1$; crosses refer to configurations where the neutralino is a sub-dominant dark matter component: $\Omega_{\chi} h^{2}<0.05$, i.e., $\xi<1$. The slanted solid line shows $N_{\chi}$ for $m_{\chi}=50 \mathrm{GeV}$ and for the limiting case of nonannihilating WIMPs. This line represents, for each of the two limiting cases of coherent and spin dependent domination, the actual upper limit for $N_{\chi}$ for neutralinos in the effective MSSM, due to the lower bound on the neutralino mass quoted above. The dot-dashed lines denote iso- $\delta$ contours for two representative values of $\delta$. For each pair, the upper line refers to $m_{\chi}=50 \mathrm{GeV}$ and $\xi=1$, while the lower line refers to $m_{\chi}=500 \mathrm{GeV}$ and $\xi=1$.

By comparing the scatter plot with the iso- $\delta$ lines, we can notice that the configurations with no rescaling $(\xi=1)$ are compatible with values of $\delta$ smaller than about $10^{-9}$, both for coherent and spin-dependent interactions. We remind that these are configurations with large neutralino relic abundance. In the case of configurations with $\xi<1$, we have to consider the shift of the iso- $\delta$ lines shown in Fig. 3 and the range of values for $\xi$ in the MSSM shown in Fig. 7. In this case, we obtain that the maximal effect induced by a subdominant relic neutralino is for values of $\delta$ of the order of $10^{-6}$ for the coherent case and $10^{-4}$ for the spin-dependent one.

We can therefore conclude that the maximal effects induced in the Sun by relic neutralinos are much smaller than those valid for a generic WIMP and discussed in Sec. IV.
This implies that the specific case of the neutralino has even fewer chances to be bounded by the study of solar properties.

\section{CONCLUSIONS}

Our understanding of solar physics has significantly advanced in recent times, because of remarkable improvements in helioseismology and of new data on solar neutrinos. In the present paper we have addressed the question of whether these new developments put solar physics in a position to provide constraints on the possible presence of WIMPs in the core of the Sun, for values of WIMP parameters under current exploration in WIMP searches. We summarize here our main results:

We have provided a quantitative criterium to determine whether a putative WIMP candidate could produce observable modifications of the solar structure. Namely, we have introduced the parameter $\delta$, defined as the ratio between the WIMP luminosity and the radiative luminosity at the center of the Sun [see Eq. (17)], which can be analytically calculated as a function of the WIMP-nucleon scattering cross section $\sigma_{p}$ and the number of WIMPs in the Sun $N_{\chi}$. By considering the uncertainty in the radiative opacity, one finds that $\delta \gtrsim 10^{-2}$ is a necessary (but not sufficient) condition for WIMPs to have observable effects on the Sun.

We have calculated the number of WIMPs in the Sun for a generic WIMP candidate as a function of the WIMPnucleon scattering cross section $\sigma_{p}$ and of the WIMPantiWIMP pair annihilation cross section $\sigma_{a}$, both for coherent and spin-dependent WIMP interactions. We have shown that a value $\delta \sim 10$ can be reached, if WIMP-antiWIMP annihilation is negligible and $\sigma_{p} \sim 10^{-37} \mathrm{~cm}^{2}$ (coherent scattering) or $\sigma_{p} \sim 10^{-34} \mathrm{~cm}^{2}$ (spin dependent interactions). For coherent WIMP scattering, such large values for the cross sections are excluded by direct search experiments, unless WIMPs give a subdominant contribution to dark matter of our galaxy. In this case, if one takes into account direct search bounds $\left(\xi \sigma_{p} \lesssim 10^{-41} \mathrm{~cm}^{2}\right.$, where $\xi$ is the rescaling parameter which accounts for the actual fraction of local dark matter to be ascribed to the given WIMP), one obtains $\delta \leqslant 10^{-6}$ in the assumption of no rescaling $(\xi=1)$ and $\delta$ $\lesssim 10^{-3}$ in the general case $(\xi \leqslant 1)$.

We have considered the neutralino as a specific WIMP candidate. We have calculated the number of neutralinos in the Sun for MSSM configurations compatible with present experimental and cosmological constraints. For each configuration, we determined the rescaling parameter $\xi$ by the standard rescaling procedure described in Eq. (27). In the case of no rescaling $(\xi=1)$, we obtained $\delta \leqslant 10^{-9}$. For subdominant neutralinos $(\xi \leqslant 1)$, we obtained $\delta \leqslant 10^{-6}$ for configurations in which the capture rate is dominated by coherent scattering and $\delta \leqq 10^{-4}$ for configurations in which the dominant contribution is due to spin dependent interactions.

The previous points already show that solar physics is not competitive with direct experiments in a large part of the WIMP parameter space under current exploration in WIMP searches. In order to complete our analysis and to understand whether some information could be obtained from the present helioseismic and solar neutrino data, we have con- 
structed solar models, for WIMP masses above $30 \mathrm{GeV}$, choosing the value of the WIMP parameters which maximize the effect of WIMPs on the Sun $(\delta \sim 10)$. As a result of the presence of WIMPs in the Sun, we obtained variations of the sound speed profile and of the ${ }^{8} \mathrm{~B}$ neutrino flux which are within the current experimental uncertainties. The smallness of the effects is essentially due to the the smallness of the WIMP extension region which, for WIMPs with masses larger than tens of $\mathrm{GeV}$, is $r_{\chi} \leqslant 0.02 R_{\odot}$.

In conclusion, no constraints can be derived at present from solar physics for WIMPs with masses above $30 \mathrm{GeV}$. Our conclusions are at variance with results derived in Refs. $[29,30]$. The origins of these disagreements have been elucidated in the present paper.

\section{APPENDIX}

We give here for completeness the expression of the factor $S_{i}$, introduced in Eq. (25), as derived from Ref. [35]. It is given by

$$
S_{i}=\frac{\left\langle F_{i}\right\rangle_{i}}{\langle\phi\rangle_{i} \xi(\infty)},
$$

where the brackets indicate an average over the mass density profile $\rho_{i}(r)$ of the $i$ th nucleus in the Sun:

$$
\langle f\rangle_{i} \equiv \frac{1}{M_{i}} \int_{0}^{R_{\odot}} 4 \pi r^{2} d r \rho_{i}(r) f(r) .
$$

All the quantities are defined in Sec. III with the exception of $F_{i}$, which is given by

$$
\begin{aligned}
F_{i}= & \frac{\bar{v}^{2}}{v_{e s c}^{2}} \frac{1}{3 b \eta}\left\{\left[\chi(-\hat{\eta}, \hat{\eta})-\chi\left(\hat{A}_{-}, \hat{A}_{+}\right)\right] \frac{\exp \left(-a \hat{\eta}^{2}\right)}{(1+a)^{1 / 2}}\right. \\
& -\left[\chi(-\check{\eta}, \check{\eta})-\chi\left(\check{A}_{-}, \check{A}_{+}\right)\right] \frac{\exp \left(-b \check{\eta}^{2}\right)}{(1+b)^{1 / 2}} \\
& \left.\times \exp \left[-(a-b) A^{2}\right]\right\} .
\end{aligned}
$$

The definition of the quantities $a, b, \eta, A, \hat{A}_{+,-}, \check{A}_{+,-}, \hat{\eta}$, and $\check{\eta}$ may be found in Ref. [35].
[1] See, for instance, Y. Sofue and V. Rubin, astro-ph/0010594; S.L. Bridle et al., astro-ph/9903472.

[2] See, for instance, S. Esposito, G. Mangano, G. Miele, and O. Pisanti, Nucl. Phys. B568, 421 (2000); E. Lisi, S. Sarkar, and F.L. Villante, Phys. Rev. D 59, 123520 (1999); G. Fiorentini, E. Lisi, S. Sarkar, and F.L. Villante, ibid. 58, 063506 (1998).

[3] See, for instance, N. Fornengo, Nucl. Phys. B (Proc. Suppl.) 110, 26 (2002); hep-ph/0201156; A. Bottino and N. Fornengo, hep-ph/9904469.

[4] For a review of WIMP direct searches see, for instance, A. Morales, Nucl. Phys. B (Proc. Suppl.) 110, 39 (2002).

[5] A. Bottino, F. Donato, G. Mignola, S. Scopel, P. Belli, and A. Incicchitti, Phys. Lett. B 402, 113 (1997).

[6] P. Belli, R. Bernabei, A. Bottino, F. Donato, N. Fornengo, D. Prosperi, and S. Scopel, Phys. Rev. D 61, 023512 (2000).

[7] P. Belli, R. Cerulli, N. Fornengo, and S. Scopel, Phys. Rev. D 66, 043503 (2002).

[8] DAMA/NaI Collaboration, R. Bernabei et al., Phys. Lett. B 424, 195 (1998); 450, 448 (1999); 480, 23 (2000).

[9] A.K. Drukier, K. Freese, and D.N. Spergel, Phys. Rev. D 33, 3495 (1986); K. Freese, J.A. Frieman, and A. Gould, ibid. 37, 3388 (1988).

[10] A. Bottino, F. Donato, N. Fornengo, and S. Scopel, Phys. Lett. B 423, 109 (1998); Phys. Rev. D 59, 095003 (1999); 59, 095004 (1999); 62, 056006 (2000); 63, 125003 (2001).

[11] R. Arnowitt and P. Nath, Phys. Rev. D 60, 044002 (1999); E. Accomando, R. Arnowitt, B. Dutta, and Y. Santoso, Nucl. Phys. B585, 124 (2000); R. Arnowitt, B. Dutta, and Y. Santoso, hep-ph/0008336; J.L. Feng, K. Matchev, and F. Wilczek, Phys. Lett. B 482, 388 (2000); E. Gabrielli, S. Khalil, C. Muñoz, and E. Torrente-Lujan, Phys. Rev. D 63, 025008 (2001); D.G. Cerdeño, S. Khalil, and C. Muñoz, hep-ph/0105180; D.G. Cer- deño, E. Gabrielli, S. Khalil, C. Muñoz, and E. Torrente-Lujan, Nucl. Phys. B603, 231 (2001); M.E. Gómez and J.D. Vergados, Phys. Lett. B 512, 252 (2001).

[12] A. Gould and G. Raffelt, Astrophys. J. 352, 654 (1990).

[13] D.N. Spergel and W.H. Press, Astrophys. J. 294, 663 (1985).

[14] A. Gould and G. Raffelt, Astrophys. J. 352, 669 (1990).

[15] R. Gilliland, J. Faulkner, W.H. Press, and D.N. Spergel, Astrophys. J. 306, 703 (1986).

[16] D. Dearborn, K. Griest, and G. Raffelt, Astrophys. J. 368, 626 (1991).

[17] A. Bouquet and P. Salati, Astron. Astrophys. 217, 270 (1989); J. Kaplan, F. Martin de Volnay, C. Tao, and S. Turck-Chièze, Astrophys. J. 378, 315 (1991).

[18] S. Degl'Innocenti, W.A. Dziembowski, G. Fiorentini, and B. Ricci, Astropart. Phys. 7, 77 (1997).

[19] W.A. Dziembowski, A.A. Pamiatnykh, and R. Sienkiewicz, Mon. Not. R. Astron. Soc. 249, 602 (1991).

[20] J. Christensen-Dalsgaard, D.O. Gough, and M.J. Thompson, Astrophys. J. 378, 413 (1991).

[21] F.L. Villante, G. Fiorentini, and E. Lisi, Phys. Rev. D 59, 013006 (1999).

[22] G. Fiorentini, F.L. Villante, and B. Ricci, hep-ph/0109275.

[23] G.L. Fogli, E. Lisi, D. Montanino, and A. Palazzo, Phys. Rev. D 64, 093007 (2001).

[24] G.L. Fogli, E. Lisi, D. Montanino, and A. Palazzo, Phys. Rev. D 65, 117301 (2002).

[25] SNO Collaboration, Q.R. Ahmad et al., Phys. Rev. Lett. 87, 071301 (2001).

[26] SuperKamiokande Collaboration, S. Fukuda et al., Phys. Rev. Lett. 86, 5651 (2001).

[27] SNO Collaboration, Q.R. Ahmad et al., Phys. Rev. Lett. 89, 011301 (2002) 
[28] G. Fiorentini and B. Ricci, Phys. Lett. B 526, 186 (2002).

[29] I.P. Lopes, J. Silk, and S.H. Hansen, Mon. Not. R. Astron. Soc. 331, 361 (2002).

[30] I. Lopes and J. Silk, Phys. Rev. Lett. 88, 151303 (2002).

[31] K. Griest and D. Seckel, Nucl. Phys. B283, 681 (1987).

[32] W.H. Press and D.N. Spergel, Astrophys. J. 296, 679 (1985).

[33] F. Ciacio, S. Degl'Innocenti, and B. Ricci, Astron. Astrophys., Suppl. Ser. 123, 449 (1997).

[34] J.N. Bahcall and M.H. Pinsonneault, Rev. Mod. Phys. 64, 885 (1992).

[35] A. Gould, Astrophys. J. 321, 571 (1987).

[36] S. Basu, M.H. Pinsonneault, and J.N. Bahcall, Astrophys. J. 529, 1084 (2000).

[37] S. Turck-Chieze et al., Sol. Phys. 200, 323 (2001).

[38] S. Turck-Chieze et al., Astrophys. J. Lett. 555, L69 (2001).
[39] J.N. Bahcall, M.H. Pinsonneault, and S. Basu, Astrophys. J. 555, 990 (2001).

[40] G.L. Fogli, E. Lisi, A. Palazzo, and F.L. Villante, Phys. Rev. D 63, 113016 (2001).

[41] A. Bottino, F. Donato, N. Fornengo, and S. Scopel, Phys. Rev. D 63, 125003 (2001); A. Bottino, N. Fornengo, and S. Scopel, in Proceedings of COSMO-01, Rovaniemi, Finland, 2001 [Nucl. Phys. B (Proc. Suppl.) 110, 76 (2002)].

[42] T.K. Gaisser, G. Steigman, and S. Tilav, Phys. Rev. D 34, 2206 (1986).

[43] A. Bottino, V. de Alfaro, N. Fornengo, G. Mignola, and M. Pignone, Astropart. Phys. 2, 67 (1994).

[44] J.N. Bahcall and M.H. Pinsonneault, Rev. Mod. Phys. 67, 781 (1995). 